\title{
Construcción de paz y conflictos region- ales en los países insulares del Pacífico: en busca del buen gobierno
}

\author{
Yoko Ogashiwa
}

\begin{abstract}
Following the coups d'états in the Fiji and Solomon Islands in 2000, the Pacific Islands Forum has been constantly promoting multilateral initiatives in order to mediate and prevent further conflicts in the region. These efforts included the support of "good governance" practices. However, because of their alien character, good governance initiatives have not been exempted from tensions with the traditional practices of governance. This paper explains the ethnic character of the conflicts in the Pacific Islands, placing a particular emphasis on the gradual adoption by the Forum's Members of the above-mentioned alien concepts and on the criticism made by some prominent activists -native to the region- with regard to the concepts.
\end{abstract}

\section{Introducción}

Dos golpes de estado estallaron, uno tras otro, en los países insulares del Pacífico en el año 2000, uno en Fiji en mayo, y el otro en las Islas Salomón en junio. Estos incidentes llevaron al Foro de las Islas del Pacífico (en lo sucesivo, el Foro), una organización regional establecida en 1971, a adoptar la Declaración de Biketawa en su reunión anual en octubre de 2000, que define los principios rectores y cursos de acción que deben ser adoptados por el Foro en caso de crisis políticas en la región. Luego de la adopción de la Declaración de Biketawa, el Foro cambió su postura no interventora, para intervenir en los conflictos de la región. ${ }^{1}$

El Foro se involucró especialmente en el conflicto de las Islas Salomón, que fueron categorizadas como un "Estado débil" en Melanesia (Instituto de Política Estratégica de Australia, 2003), y donde algunos académicos han afirmado que está dándose un proceso de "africanización" (Reilly y Wainwright, 2005:125). 
Tras el envío de la misión de observación electoral en diciembre de 2001, se envió un grupo de personas eminentes a las Islas Salomón en junio de 2002. Por petición del gobierno de las Islas Salomón, se realizó una intervención militar regional en julio de 2003 a cargo del personal militar y de policía de Australia, Nueva Zelanda, Papua y Nueva Guinea, Fiji y Tonga. Esto fue seguido por el establecimiento de la Misión de Asistencia Regional a las Islas Salomón como un organismo de control para intervención regional. ${ }^{2}$ La intervención del Foro en el conflicto de las Islas Salomón atrajo la atención como primer caso basado en la Declaración de Biketawa.

Si bien ha intervenido directamente en el conflicto, el Foro ha tratado de tener un buen gobierno en la región como parte del proceso regional de construcción de paz, incluso antes de adoptar la Declaración de Biketawa. ¿De qué manera ha tratado el Foro de tener un buen gobierno en la región? Y, ¿hasta qué punto son estos intentos del Foro regional efectivos para la construcción de paz en la región? El propósito de este artículo es examinar los intentos del Foro para formar un buen gobierno y analizar sus aportes para la construcción de paz en la región de los países insulares del Pacífico. En primer lugar, el artículo considera las características de los conflictos en los estos países, seguido por un panorama general del compromiso del Foro con el buen gobierno. Más adelante se pasa a explorar la forma en que el Foro ha tratado de formar un buen gobierno en la región. En la última sección, se discute la eficacia de la tentativa del Foro para construir la paz en la región.

\section{Características de los conflictos en los países insulares del Pacífico}

Cuando pensamos en los conflictos de los países insulares del Pacífico es necesario tener en cuenta dos factores. El primero de ellos es el origen étnico, dado que las islas del Pacífico conforman países muy diversos, los cuales están divididos en tres sub-regiones: Polinesia, Melanesia y Micronesia. En cada subregión existen diferencias en los distintos niveles, tales como país, isla, distrito y aldea. Por ejemplo, en los países insulares del Pacífico se hablan diferentes idiomas. Se dice que en Melanesia se hablan más de 1200 idiomas indígenas, es decir que aproximadamente una cuarta parte de las lenguas del mundo se hablan allí (Reilly y Wainwright, 2005: 127). La mayoría de los conflictos en los países insulares del Pacífico se han dado entre los diferentes grupos étnicos y, en el caso de las Islas Salomón, el conflicto estalló entre los residentes de Guadalcanal y los invasores de Malaita. También, en el caso de Bougainville, en Papua y Nueva Guinea, los habitantes de Bougainville lucharon por independizarse de Papua y Nueva Guinea. Fiji es otro ejemplo típico de conflicto entre grupos

2 Sobre la intervención del Foro en el conflicto de las Islas Salomón, véase Ogashiwa 2004. 
étnicos: los indígenas fijianos vs. los indo-fijianos que son descendientes de obreros inmigrantes.

Sin embargo, tenemos que señalar que cada grupo étnico no siempre es homogéneo internamente. Es bastante común que existan diferencias dentro del grupo étnico. Por ejemplo, los fijianos se dividen a lo largo de una línea regional, a un lado las islas orientales influenciadas por la Polinesia y, al otro, las islas occidentales influenciadas por la Melanesia. A lo largo de la línea de un sistema social tradicional se dividen en varias poblaciones desde los mataqali (población de terratenientes), los yavusa (clan compuesto de una serie de mataqali) hasta los vanua (mayor población social y política compuesta de una serie de yavusa). Los indofijianos también están divididos en varios sub-grupos, como los hindúes, los musulmanes, los cristianos y los sikh.

Aunque existen diferencias en el seno del grupo étnico, la gente tiende a estar vinculada y comprometida con los conflictos. Esto se debe al segundo factor de los conflictos, a saber, las disparidades políticas y económicas. El conflicto en Bougainville nos da un ejemplo de esto: sus habitantes se han fragmentado en 19 grupos lingüísticos diferentes (Reilly, 2004: 488). Sin embargo, la situación ha cambiado desde que se empezó a trabajar la minería del cobre en Bougainville en 1964. Luego de que Papua y Nueva Guinea alcanzara la independencia en 1975, el cobre de Bougainville se convirtió en un producto importante que sostuvo la economía nacional. Desde 1970 hasta la década de 1980, la minería del cobre representó el 44\% de las exportaciones de Papua y Nueva Guinea. Si bien Bougainville hizo una contribución sustancial a la economía nacional de Papua y Nueva Guinea, el descontento con la distribución de los beneficios de la minería se hizo notar entre los habitantes de Bougainville (McDougall ,2004: 343-344) y en 1989 un grupo de de personas oriundas de Bougainville sabotearon la mina. El gobierno de Papua y Nueva Guinea envió las fuerzas militares a Bougainville, empezando así el conflicto armado entre el ejército y las fuerzas secesionistas locales llamadas Ejército Revolucionario de Bougainville (BRA). Aunque no se puede decir que el BRA obtuvo el apoyo de una amplia gama de la población local, sus pretensiones de independizarse de Papua y Nueva Guinea respondieron al sentimiento compartido de los habitantes de Bougainville de que eran explotados económicamente por el gobierno nacional (McDougall, 2004: 343-344). Así pues, se generó "la identidad pan-Bougainvilense" (Reilly, 2004: 488).

Sin embargo, es necesario señalar una vez más que los conflictos no sólo son causados por disparidades políticas y económicas, sino también por el origen étnico. Así las disparidades políticas y económicas logren que el conflicto sea más grave, su base es el factor étnico. El conflicto en Bougainville demostró que 
tales disparidades en torno a la minería de cobre fueron un factor importante, pero al mismo tiempo cabe señalar que la heterogeneidad de Bougainville y de Papua y Nueva Guinea también influyó en el conflicto. Aunque los habitantes de Bougainville estaban vinculados étnicamente a la población de la provincia occidental de las Islas Salomón, Bougainville se incorporó en el territorio de Papua y Nueva Guinea basándose en las fronteras establecidas en la época colonial. Por lo tanto, los habitantes de Bougainville han sido considerados por los demás, y por ellos mismos, como distintos de otros grupos étnicos en Papua y Nueva Guinea. Reilly citó su notorio color de piel como "pueblo negro azabache", como un índice de carácter distintivo de los habitantes de Bougainville (Reilly, 2004: 488), lo cual ilustra claramente el factor étnico en el conflicto en Bougainville. Por lo tanto, es difícil pensar en los factores étnico y políticoeconómico por separado en los conflictos de los países insulares del Pacífico, ambos están estrechamente entrelazados. Es exactamente en este sentido que podemos categorizar los conflictos en estos países como "conflictos étnicos".

\section{El Foro de las Islas del Pacífico y el buen gobierno}

Fue en la Declaración de Visión del Foro del Pacífico Sur, adoptada en la reunión anual en 1995, que el Foro se refirió por primera vez al buen gobierno. En el proceso de presentar una visión para el fortalecimiento de la cooperación regional del Foro para los 25 años siguientes, la declaración mencionó el buen gobierno como parte de la misma (Secretaría del Foro del Pacífico Sur, 1995) y, dos años más tarde, el Foro señaló el buen gobierno como parte de la cooperación en seguridad regional. En la reunión anual, el Foro aprobó la Declaración de Aitutaki sobre Cooperación a la Seguridad Regional e hizo énfasis en el buen gobierno como uno de los principios rectores que rigen la cooperación para la seguridad en la región, junto con el desarrollo sostenible y la cooperación internacional, incluida la diplomacia preventiva (Secretaría del Foro del Pacífico Sur, 1997a). Además, como se mencionó anteriormente, la Declaración de Biketawa fue adoptada en la reunión anual del Foro en el año 2000 y se incluyó el "compromiso con el buen gobierno" en la declaración, como uno de los principios rectores en el caso de crisis políticas en la región (Secretaría del Foro de los Países Insulares del Pacífico, 2000a). "El compromiso con el buen gobierno" también se menciona en la Declaración de Nasonini sobre Seguridad Regional, aprobada en la reunión anual en 2002, como "una estrategia clave fundamental para hacer frente a algunas de las difíciles y delicadas cuestiones que subyacen a la causa de tensión y conflicto en la región" (Secretaría del Foro de Islas del Pacífico, 2002a). 
Entonces, ¿cómo ha definido el Foro el concepto de buen gobierno? Se puede decir que el buen gobierno para el Foro está integrado por tres elementos: la rendición de cuentas, la transparencia y la efectiva participación del público en la toma de decisiones (Foro de Revisión, 2000: 9). Noel Levi, Secretario General del Foro, declaró en la reunión sobre gobierno para los parlamentarios en la región del Pacífico en el año 2000, que la rendición de cuentas se trataba de "derechos de los ciudadanos, o grupos clave de amplia base en la comunidad, para hacer responsables a los funcionarios públicos del comportamiento del gobierno y que respondan a las necesidades de los ciudadanos". Acerca de la transparencia, explicó que esta reducía la incertidumbre y ayudaba a inhibir la corrupción entre funcionarios públicos y elegidos. Además, hizo hincapié en la idea de que los que rigen y los que son gobernados deben tener igual participación en el proceso de toma de decisiones (Foro de Revisión, 2000: 9).

Tomemos un caso del sector maderero como ejemplo para entender el buen gobierno en el contexto concreto de los países insulares del Pacífico. En Melanesia, las compañías del Sudeste de Asia han operado a gran escala la explotación forestal desde principios de 1980. La deforestación rápida y en gran escala, a la que se llamó "fiebre de oro verde" (Thistlethwaite y Davis, 1996:49), causó muchos problemas. Un problema grave fue la corrupción de los políticos y funcionarios públicos. Al recibir sobornos de las empresas madereras, los políticos y funcionarios públicos actuaron a favor de ellos, expidiendo licencias de tala, haciendo intocable el funcionamiento ilegal (Kabutaulaka, 2000: 95; Connell, 1997: 102, 105-106). Un incidente simbólico ocurrió cuando Ted Diro, Viceprimer Ministro y Ministro de Bosques de Papua y Nueva Guinea, fue procesado por recibir sobornos de una empresa maderera y obligado a dimitir en 1991.

La falta de una buena gestión pública, generada por el abuso de poder por parte de los políticos y funcionarios públicos como ya se mencionó, traerá una sensación de desconfianza de la gente hacia su gobierno. Esto dará lugar a ausencia de liderazgo, ocasionando que el gobierno no tenga más control sobre la ley y el orden, y provocará conflictos. Por lo tanto, el Foro ha tratado de formar un buen gobierno en la región y fortalecer la construcción de paz en la región. Sobra decir que la buena gestión pública en sí misma no es una idea propia del Foro. En primer lugar, dicha idea fue propuesta por el Banco Mundial en 1989, seguida por el Programa para el Desarrollo de las Naciones Unidas (UNDP), el Banco Asiático de Desarrollo, otros organismos de ayuda y los países donantes, y fue denominada como "condicionalidad política".

En particular, la promoción del buen gobierno por parte de la Unión Europea (UE) tuvo un impacto significativo en el Foro. Los países insulares del Pacífico, como miembros de los países ACP (África, el Caribe y el Pacífico), han gozado del especial trato comercial que ofrece la UE en el marco del Convenio 
de Lomé. No cabe duda de que las economías frágiles de los países insulares del Pacífico se han sostenido por la Convención de Lomé desde su creación en 1975. En 1995, la cuarta versión de la Convención, que entró en vigor en 1990, fue modificada en la revisión intermedia para incluir el buen gobierno como objetivo particular de las actividades de cooperación en virtud de la Convención, junto con el reconocimiento y la aplicación de los principios democráticos y de consolidación del Estado de Derecho (Arts y Byron, 1997: 83-84). ${ }^{3}$ Esto significó que los países insulares del Pacífico fueran sometidos a la presión directa de comprometerse con el buen gobierno y, en tales circunstancias, el Foro aprobó y promovió tal idea. Como se ilustra arriba, el buen gobierno es originalmente una "idea exógena" al Foro. ${ }^{4}$ ¿Cómo ha tratado el Foro de encarnar esa "idea exógena" del buen gobierno en los países insulares del Pacífico como parte de la construcción de paz regional? La siguiente sección examinará la manera en que el Foro ha tratado de formar un buen gobierno en la región.

\section{Gobierno desde arriba}

Los intentos del Foro para formar buen gobierno en la región se han llevado a cabo en dos direcciones. Una de éstas es el intento de formar "buen gobierno desde arriba", es decir, infiltrar el buen gobierno entre los parlamentarios y funcionarios públicos en los países insulares del Pacífico. Los intentos de "buen gobierno desde arriba" se iniciaron con la aprobación de los "ocho principios de rendición de cuentas" como parte del Plan de acción económico en la primera reunión del Foro de Ministros de Economía en julio de 1997 y, dos meses más tarde, tales principios se aprobaron oficialmente en la reunión anual del Foro. Estos principios consistían en las "mejores prácticas para la rendición pública de cuentas, basados en los conceptos de apertura con la información del Gobierno y el escrutinio público sobe el desempeño de los gobiernos y los funcionarios públicos" (Secretaría del Foro del Pacífico Sur, 1997b). Los puntos clave de los "ocho principios de la rendición de cuentas" son: la transparencia en la gestión fiscal de la economía, la rendición de cuentas del gobierno en cuanto a sus recursos financieros y la forma en que estos se han utilizado, y el refuerzo de la tradición del sistema de banca central independiente (Foro del Pacífico Sur Secretaría, 2001a). En julio de 1998, la Reunión de Ministros de Economía del Foro adoptó el Código de Principios y Buenas Prácticas de Transparencia Fiscal definido por el Fondo Monetario Internacional como un modelo para

3 El buen gobierno se ha mencionado como "dimensión política” en el Acuerdo de Cotonú firmado en el año 2000 como sucesor de la cuarta versión de la Convención de Lomé.

4 Haciendo uso de la metáfora, Peter Larmour llamó instituciones "transferidas" a las de los países insulares del Pacífico, "flores del exranjero" a la democracia y a la reforma del sector público, (Larmour, 2005). 
complementar los "ocho principios de rendición de cuentas" (Secretaría del Foro del Pacífico Sur, 1998).

Estos intentos también han sido llevados a cabo mediante el establecimiento del Foro de Ponentes de las Islas del Pacífico, ${ }^{5}$ compuesto por los ponentes de parlamentos de los países miembros del Foro. Este Foro se creó en 2001 tras el cierre de los parlamentos durante el golpe en Fiji y las Islas Salomón en el año 2000. Se trata de un organismo regional "para discutir la manera en que los parlamentos del Pacífico continuarán mejorando la calidad del gobierno en el marco de la democracia parlamentaria" (Secretaría del Foro de Islas del Pacífico, 2003a). En la reunión inaugural, el Foro de Ponentes de las Islas del Pacífico aprobó el Plan de Acción Regional para las legislaturas de los países insulares como "principios fundamentales de las mejores prácticas para orientar el desarrollo legislativo en todos los países insulares del Foro" (Secretaría del Foro de Islas del Pacífico, 2001b).

Sin embargo, los intentos para formar el "buen gobierno desde arriba" sobre la base de la "idea exógena" han ido cambiando. La primera aparición de cambio se muestra en el Foro de principios de un buen liderazgo, aprobado en la reunión anual del Foro en 2003. El Foro de principios de un buen liderazgo, modelo de código para la toma de decisiones sobre cómo actuar al mismo tiempo que se mantienen cargos públicos, se originó en el taller sobre códigos de liderazgo en 2002, que fue organizado por la Secretaría del Foro, el Programa para el Desarrollo de las Naciones Unidas y la Secretaría del Commonwealth (Secretaría del Foro de las Islas del Pacífico, 2002b). Está comprendido por nueve principios, tales como "el respeto de la ley y el sistema de gobierno", "respeto a las personas en cuyo nombre los dirigentes ejercen el poder" y "economía y eficiencia".

Aunque el Foro de principios de un buen liderazgo se basa fundamentalmente en la "idea exógena", es notable que "el respeto de los valores culturales, costumbres y tradiciones" se incluyó como uno de los principios (Secretaría del Foro de Islas del Pacífico, 2003b). Éste no fue incluido en los "ocho principios de rendición de cuentas" precedentes. El comunicado final de la reunión anual del Foro anotó que el Foro de principios de un buen liderazgo tuvo en cuenta "los valores tradicionales del Pacífico y del Foro de Líderes, de compromiso con los principios contenidos en la Declaración de Biketawa" (Secretaría del Foro de Islas del Pacífico, 2003c). En definitiva, el Foro de principios de un buen liderazgo considera "los valores tradicionales del Pacífico", al tiempo que se basa fundamentalmente en la "idea exógena".

5 Se cambió el nombre a Conferencia de Oficiales Presidentes del Foro, celebrada en 2002, y luego a Conferencia de Oficiales Presidentes y Empleados en 2005. 
Las consideraciones del Foro de "los valores tradicionales del Pacífico" en los intentos para formar el "buen gobierno desde arriba" fue, obviamente, más evidente en la declaración dada por Greg Urwin, Secretario General del Foro, en el Foro de Conferencia para oficiales presidentes en 2004. Urwin mencionó que los dirigentes del Foro recibieron una evaluación del Foro y sus actividades, llevadas a cabo por el eminente grupo de personas, y afirmó que los objetivos enumerados en la evaluación, incluyendo el buen gobierno, deben llevarse a cabo en el contexto de la protección y el desarrollo de la identidad cultural de la región. Luego, afirmó que la gobernabilidad no es un valor externo impuesto desde ultramar e hizo hincapié en lo siguiente:

Hay mucho que puede y debe aprenderse de nuestras comunidades tradicionales en materia de ejercicio de la autoridad, ¿de qué otra manera pueden estas comunidades haber desarrollado un complejo conjunto de orientaciones culturales y sociales, mucho antes de la introducción de la alfabetización u otros aspectos de la sociedad que reconocemos como moderna? (Secretaría del Foro de Islas del Pacífico 2004a).

Al mismo tiempo, Urwin preguntó a los voceros y funcionarios de los parlamentos, como "campeones del gobierno", quién ayudaría a crear un entorno en el que pudiera prosperar la gobernabilidad, para observar el buen gobierno basado en la "idea exógena", como la rendición de cuentas. No obstante, cabe señalar que el Foro está tratando de redefinir el buen gobierno en relación con los "valores tradicionales del Pacífico", como lo demuestra el hecho de que Urwin haya pedido "buen gobierno en el Pacífico" en la declaración (Secretaría del Foro de Islas del Pacífico, 2003c).

Una de las razones de la redefinición del Foro sobre el buen gobierno fue la reacción a la buena gestión pública basada en la "idea exógena" entre los dirigentes políticos de los países insulares del Pacífico. Por ejemplo, Berenado Vunibobo, Ministro de Hacienda de Fiji, quien asistió a la Reunión de Ministros de Economía del Foro en 1997, donde se aprobaron los "ocho principios de rendición de cuentas", criticó la idea de buen gobierno tildándola de una "moda" diciendo: "En un momento se oye el sistema internacional hablar de recursos humanos y desarrollo y después de un tiempo algo más se perfila como lo mayor y más importante en los últimos años, el buen gobierno (Negocios Insulares, 1997: 35)". El Foro tuvo que considerar "los valores tradicionales del Pacífico" a fin de aplacar la reacción contra el buen gobierno basado en la "idea exógena" entre los dirigentes políticos, cuyos intentos de "buen gobierno desde arriba" estuvieron en la mira. 
En segundo lugar, y aun más importante, surgió la idea de examinar de nuevo y reevaluar los "valores tradicionales del Pacífico" en el contexto del buen

gobierno en la discusión sobre este último. Este punto será discutido con mayor precisión en la última sección. Cabe señalar aquí una vez más que los intentos del Foro para el "buen gobierno desde arriba", originado en la "idea exógena", apuntan hacia una nueva dirección de reinterpretación de la buena gestión pública desde la perspectiva de los "valores tradicionales del Pacífico".

\section{Gobierno desde abajo}

Así como se han realizado intentos de "buen gobierno desde arriba", el Foro ha llevado a cabo intentos para "el buen gobierno desde abajo". Este ha sido el intento de facilitar a la "sociedad civil" su participación en el proceso de toma de decisiones en la región. Fue en la Secretaría del Foro y en el Marco de Consulta Política de las Organizaciones No Gubernamentales, aprobado en el año 2000, que el Foro hizo el primer intento para "el buen gobierno desde abajo". Antes de esto, el Foro se había referido a la necesidad de cooperación con la "sociedad civil" en la Declaración de Visión del Foro del Pacífico Sur 1995, el Foro de Revisión de Plan de Acción Económica y el comunicado final de la reunión anual del Foro en 1999 (Secretaría del Foro de Islas del Pacífico, 2002c). En la Secretaría del Foro y en el Marco de Consulta Política de las Organizaciones No Gubernamentales, el Foro consideró oficialmente a las Organizaciones No Gubernamentales (ONG) como socios de consulta en el proceso de toma de decisiones de la región.

La Secretaría del Foro y el Marco de Consulta Política de las Organizaciones No Gubernamentales dividieron a las ONG en dos grupos, esto es, en "sociedad civil" y "sector empresarial", y establecieron reuniones anuales de diálogo para debatir cuestiones de política. Los criterios para la participación en las reuniones incluyeron "ser una organización no gubernamental legalmente constituida enfocada en el Pacífico y una membrecía como miembro del Foro; que trabaje en algún área clave de las actividades de la Secretaría y que también colabore activamente con los gobiernos miembros o asociados" (Secretaría del Foro de Islas del Pacífico, 2000b). El Foro facilitó información a las organizaciones no gubernamentales sobre cuestiones de política, a través de las tecnologías de la información y las alentó a asistir en calidad de observadores a las reuniones del Foro adecuadas y específicas de su respectivo sector (Ibíd.).

Los intentos para "el buen gobierno desde abajo" se fortalecieron aún más con la sustitución de la Secretaría del Foro y el Marco de Consulta Política de las Organizaciones No Gubernamentales por el Marco para el Compromiso con Actores No Estatales de la Región Pacífica en el año 2002. Al redefinir "los 
actores no estatales" como socios de consulta, el Marco para el Compromiso con Actores No Estatales de la Región Pacífica abrió espacio para que la "sociedad civil" participara en el proceso de toma de decisiones a través varios mecanismos, como el establecimiento de reuniones de consulta bienales, la concesión del estatus de observador en las reuniones del Foro de reuniones regionales de alto nivel, la admisión en grupos focales para el desarrollo de documentos para la formulación de la política y la participación en el Grupo de Trabajo del Consejo de Organizaciones Regionales del Pacifico. ${ }^{6}$ (Secretaría del Foro de Islas del Pacífico, 2002c).

Además, el Foro hizo también intentos para "el buen gobierno desde abajo", invitando a la "sociedad civil" a las consultas sobre el desarrollo del Plan del Pacífico, que fue un plan de acción para la aplicación de los objetivos futuros, para el fortalecimiento de la cooperación regional y la integración del Foro. Once "actores no estatales" fueron invitados a las consultas y discutieron la cuestión con la Secretaría del Foro (Secretaría del Foro de Islas del Pacífico, 2004b). Además, un representante de la "sociedad civil" fue empleado como vínculo con las organizaciones de la "sociedad civil" en la Oficina del Plan del Pacífico de la Secretaría del Foro en 2005, aunque el trabajo se extendió a contrato por sólo seis meses. Se trataba del primer caso en que se hacía el nombramiento de un representante de la "sociedad civil" por parte del Foro (Secretaría del Foro de Islas del Pacífico, 2005).

Los intentos del Foro fueron transformados con la aprobación de la Política de Estatus, de Consulta y de Acreditación de la Secretaría del Foro de Islas del Pacífico y los actores no estatales de la región del Pacífico en 2007. En virtud de la Política, el Foro estableció el Estatuto del Comité Consultivo, integrado por representantes del anterior, actual y futuro Foro de Presidentes, más un representante de la Secretaría del Foro, e hizo más preciso el estatus consultivo de "los actores no estatales" (Política sobre el Estatus Consultivo y Acreditación entre la Secretaría del Foro de las Islas del Pacífico y los Actores no estatales de la región del Pacífico, 2007).

Por supuesto, iguales a los intentos para "el buen gobierno desde arriba", los intentos para "el buen gobierno desde abajo" se basaron originariamente en la "idea exógena" defendida por los proveedores de ayuda. En particular, se puede decir que el Acuerdo de Cotonú aprobado en 2000 entre la UE y los países ACP, de los cuales los países insulares del Pacífico son miembros, ha

6 El Consejo de Organizaciones Regionales del Pacífico está integrado por la Secretaría del Foro, la Secretaría de la Comunidad del Pacífico (anteriormente Comisión del Pacífico Sur), el Foro de Agencias de Pesca, el Programa Ambiental Regional del Pacífico Sur, la Universidad del Pacífico Sur, la Escuela de Medicina de Fiji, el Programa de Desarrollo de las Islas del Pacífico, la Comisión de Geociencias Aplicadas del Pacífico Sur, la Organización de Turismo del Pacífico Sur, y la Junta para la Evaluación Educativa del Pacífico Sur. 
influido directamente en los intentos Foros por formar "buen gobierno desde abajo". El Acuerdo de Cotonú requería que los países insulares del Pacífico consultaran a "los actores no estatales" en el desarrollo de la política, especialmente en lo concerniente a la ayuda de la UE, y la utilización de sus servicios en la implementación de programas posteriores (Secretaría del Foro de Islas del Pacífico, 2002c).

¿Cuáles son, entonces, "los actores no estatales" que tienen el mandato de impulsar el "buen gobierno desde abajo"? Por ejemplo, la Conferencia de Iglesias del Pacífico y el Consejo de Sindicatos de Comercio Oceánico y del Pacífico Sur, han participado no sólo en lo que concierne a ellos específicamente, sino también en cuestiones más amplias como el desarrollo, el género y los asuntos nucleares en la región. Asimismo, el Centro de Recursos de Asuntos del Pacífico, establecido originalmente como una secretaría del Movimiento Pacífico Independiente y Libre de Armas Nucleares, ha expandido su interés a cuestiones más amplias, incluyendo los derechos humanos y el buen gobierno, y el desarrollo humano sostenible. Puede ser también que la Fundación Internacional de los Pueblos del Pacífico Sur haya participado en cuestiones de desarrollo, junto con el buen gobierno y la democracia, y el Centro para la Crisis de la Mujer de Fiji, que se especializa en cuestiones de género. Así, "los actores no estatales" de la región, las sucursales regionales de organizaciones no gubernamentales del mundo, como Paz Verde y el Fondo Mundial para la Naturaleza, tienen también la tarea de impulsar el "buen gobierno desde abajo", junto con el Foro.

Así, en la región ha existido una importante base que apoya los intentos del Foro de "buen gobierno desde abajo". Sin embargo, cabe recordar que estos "actores no estatales" son los que cumplen con lo que la "idea exógena" requiere. El Foro los reconoce como actores que han cumplido con los criterios de "agentes no estatales", tales como "estar organizados y dirigidos en forma democrática y transparente", basándose en la "idea exógena" defendida por los donantes de ayuda. En otras palabras, "los actores no estatales", como socios para impulsar los intentos de "buen gobierno desde abajo", son seleccionados cuidadosamente por el Foro de acuerdo con la "idea exógena".

\section{Argumentos sobre el buen gobierno y la paz regional}

Como parte del proceso de construcción de paz regional, el Foro ha tratado de formar buen gobierno en la región "desde arriba" y "desde abajo". ¿En qué medida estos intentos son efectivos para la paz regional? Podríamos señalar la respuesta en la discusión sobre el buen gobierno, en parte, como se menciona en la sección anterior. 
Un interesante argumento lo presenta Hilda Lini ${ }^{7}$ de Vanuatu, quien trabajó como directora del Centro de Recursos de Asuntos del Pacífico de 2001 a 2004. Lini afirma que la constitución y las leyes son la promoción de los modelos occidentales de gobierno sin basarse en los sistemas existentes, y hace un llamado bien sea a la integración de los sistemas de gobierno tradicionales y occidentales, o a un nuevo modelo basado en ambos (Pacific Magazine, 2001: 8). Según ella, la gestión pública en el concepto indígena está vinculada a un sistema de creencias que supervisa y controla la coexistencia pacífica de todos y de todo lo que comparte el "multidimensional mundo natural en el que viven”. El líder asume la responsabilidad de indemnizar a quien sea necesario por el incumplimiento de la paz en nombre de los miembros de su comunidad. Ella llama al "gobierno indígena" como "Sistema Melanesiano de Democracia", el cual ofrece libre participación a todos los miembros de la comunidad y es abierto en sus decisiones. Así mismo, subraya que debe considerarse seriamente la adopción de "modelos de gobierno indígenas" como base para los gobiernos de los países insulares del Pacífico, y tomar como préstamo sólo algunos aspectos de los sistemas de gobierno extranjero que contribuyan a la defensa de la propiedad colectiva de coexistencia pacífica (Lini, 2003).

El argumento de Lini tiene mucho en común con la idea de re-evaluación de "los valores tradicionales del Pacífico" a los que se hacía referencia en la sección titulada "gobierno desde arriba". Se puede decir que la mayoría de las personas, incluyendo a Lini, que abogan por una nueva evaluación de "los valores tradicionales del Pacífico" son denominadas "élites modernas" con un alto nivel educativo, y que los "valores tradicionales del Pacífico" que ellos preconizan son más bien ideales, más que un hecho real. Sin embargo, un punto importante aquí es que estos argumentos que destacan los intentos para formar el buen gobierno hasta la fecha han pasado por alto "el gobierno indígena”. Como ya se ha examinado, estos argumentos han estudiado ese buen gobierno en relación con los "valores tradicionales del Pacífico".

Por otra parte, Peggy Fairbairn-Dunlop, ${ }^{8}$ quien ha participado en actividades de las ONG de la mujer en Samoa, presenta un argumento desde un ángulo diferente al de Lini. El enfoque del argumento de Fairbairn-Dunlop se sitúa

7 Hilda Lini fue la primera mujer funcionaria del programa de la Secretaría de la Comunidad del Pacífico en Nueva Caledonia, después de haber participado en el movimiento de independencia de Vanuatu, y el movimiento independiente y libre de armas nucleares. Después de regresar a Vanuatu había sido miembro del parlamento hasta el año 1998 y había estado en varios puestos ministeriales, como por ejemplo en el cargo de Ministra de Justicia. Es hermana del Primer Ministro de Vanautu Walter Lini y del actual Primer Ministro Ham Lini.

8 Peggy Fairbairn-Dunlop ha trabajado con organizaciones no gubernamentales de mujeres, incluyendo el Consejo Nacional de la Mujer en Samoa. También se desempeñó como jefe del Centro de Educación Continua de Samoa en la Universidad del Pacífico Sur, y del Programa de Estudios del Pacífico de la Universidad Victoria de Nueva Zelanda. 
en la marginación de la comunidad tradicional y de la autoridad en lugar de la re-evaluación de los "valores tradicionales del Pacífico". A ella le preocupa que la agenda del gobierno pueda conducir a una mayor centralización de la administración, restando importancia al rol de las agrupaciones basadas en la comunidad y las autoridades tradicionales, reemplazándolas por organizaciones que están vinculadas a las administraciones públicas (Fairbairn-Dunlop, 2004). Refiriéndose a la creación del Grupo de Cobertura para las organizaciones no gubernamentales de Samoa en 1998 (con el apoyo del UNDP) como la "voz oficial de las organizaciones no gubernamentales de Samoa", Fairbairn-Dunlop señala que las ONG's se dividen en dos grupos distintos, es decir, el grupo voluntario, no remunerado, independiente y con base en la comunidad "tradicional" de las organizaciones no gubernamentales, y el grupo de ONG's "profesionales", cuyo personal y programas pueden ser financiados por donantes, pero que no ostentan fuertes redes de comunidades (Fairbairn-Dunlop, 2000: 107). Según ella, las agrupaciones de base comunitaria y las autoridades que no son consistentes con la "idea exógena" preconizada por los proveedores de ayuda, tales como "la rendición de cuentas" y "la transparencia", están excluidos de los intentos de formar el buen gobierno. Sólo "los actores no estatales", que son coherentes con la "idea exógena", son seleccionados, institucionalizados y habilitados por el Foro para participar en el proceso de toma de decisiones regionales, en calidad de "sociedad civil".

Levi, secretario general del Foro, declaró en 2003 que la "sociedad civil" era a menudo un "puente" entre el gobierno y el pueblo para llegar a un régimen más participativo, en el que todas las partes interesadas se comprometieran (Secretaría del Foro, 2003d). El argumento de Fairbairn-Dunlop presenta la importante cuestión de si la "sociedad civil" representa la mayoría de la población de los países insulares del Pacífico, que viven en las comunidades tradicionales, y si realmente desempeña un papel de "puente" entre el gobierno y el pueblo para llegar a un régimen más participativo. Aunque Fairbairn-Dunlop hace hincapié en un punto diferente, tanto ella como Lini afirman que "los sistemas tradicionales" han sido pasados por alto en los intentos para formar el buen gobierno. Como pregunta Lini, ¿cómo podría ser el "gobierno indígena", al igual que "los valores tradicionales del Pacífico", realmente incorporados en los intentos del Foro para formar un buen gobierno? Además, como Fairbairn-Dunlop sostiene, ¿cómo podrían ser escuchadas las voces de las agrupaciones basadas en la comunidad y las autoridades en esos intentos? Estas son las tareas importantes para que el Foro pueda formar buen gobierno en la región.

$\mathrm{Al}$ abordar tales tareas, el Foro tiene que tener en cuenta que los conflictos en los países insulares del Pacífico son esencialmente "conflictos étnicos". Lo 
que se requiere para el Foro es la difícil tarea de estudiar "los sistemas tradicionales" en los intentos para formar el buen gobierno en la región, y al mismo tiempo, evitar el nepotismo y el etnocentrismo. En este sentido, la innovación "abierta y flexible a los sistemas tradicionales" de conformidad con el presente contexto es indispensable. Un surgimiento más eficaz de la paz en esta región depende de este punto.

\section{Bibliografía}

-Arts, Karin and Byron, Jessica (1997), “The mid-term review of the lome iv convention: heralding the future?”, en: Third world quarterly, 18 (1).

-Australian Strategic Policy Institute (2003), Ourfailing neighbour: australia and the future of solomon islands. Barton, ACT, Australian Strategic Policy Institute.

-Connell, John (1997), Papua New Guinea: The struggle for development, London, Routledge.

-Fairbairn-Dunlop, Peggy (1997), "Women NGOs within the New Governance Agenda: Are They Still Based on Alofa?", en: Elise Huffer and Asofou So'o (eds.), Governance in Samoa: Pulega i Samoa, Canberr, Asia Pacific Press and Suva, Institute of Pacific Studies, the University of the South Pacific.

(2004) "Civil society participation in the pacific" (Time to Talk: Program Ten Opinion), Radio australia, en:(http:/ /abc.net.au/timetotalk/english/opinion/Timeto talkopinion_440071.htm) (Internet, 7th July 2005),

-Forum Review (2000), "Good governance and the region” (first quarter)

-Islands Business (1997) “The economy’s new stress buster” (August)

-Kabutaulaka, Tarcisius Tara (2000), "Rumble in the jungle: land, culture and (un)sustainable logging in solomon islands", en: Hooper, Anthony (ed.), Culture and sustainable development in the pacific. Canberra, Asia Pacific Press. 
-Larmour, Peter (2005), Foreign flowers: institutional transfer and good governance

in the pacific islands, Honolulu, University of Hawai'I Press.

-Lini, Hilda (2003), "The indigenous concept of good governance”, (Time to talk: program one opinion). Radio australia (http://abc.net.au/timetotalk/english/opinion/time'To talkopinion_425873.htm), (Internet, 30th June 2005).

-McDougall, Derek (2004), "Conflicts in the southwest pacific: the relevance of new security perspectives”, en: Contemporary security policy 25 (2).

-Ogashiwa, Yoko (2003), “Chiiki, Kokka, Ethnicity: Taiheiyou Tosho Forum no Chiiki Funso eno Taiou" (in japanese) ["region, State and ethnicity: responses of the pacific islands forum to regional conflicts"], en: Matori Yamamoto, Knichi Sudo, and Shuji Yoshida (eds.), National Integration and Localism in Oceania (JCAS Area Studies Research Report 6).

, (2004) "Taiheiyou tosho forum no chiiki funso eno kanyo: solomon shoto ni okeru heiwa kochiku kara buryoku kainyu kettei made" (in japanese) "The commitment of the pacific islands forum to regional conflicts: from peace building to the decision of military intervention in solomon islands", Hiroshima Peace Science, 26.

-Pacific Islands Forum Secretariat (2000a), Press Statement 5200: "Biketawa Declaration". (http:/ / www.forumsec.org.fj/news/2000/oct06.htm), (Internet, 1st November, 2000.

, (2000b) Press Statement 5800: "Forum Secretariat Announces NGO Policy", (http://www.forumsec.org.fj/news/2000/Nov02.htm), (Internet, 29th, november, 2000).

, (2001a) Press Statement 5701, Transparency international regional meeting: statement by Mr.W. Noel Levi, CBE secretary general, Pacific Islands Forum Secretariat on "The forum eight principles of accountability; progress to date". (http:/ /www.forumsec.org.fj/news/2001/Jul05.htm), (Internet, 17th, february, 2004).

, (2001b) Twenty-Second Pacific Islands Forum: Forum Communique. (www.forumsec.org/resource/main/files/2001Communique-Nauru 16-18 Aug. pdf), (Internet, 10th, october, 2006). 
(2002b), Press Statement 1902: "Leadership code workshop". 2004, (http://www. forumsec.org.fj/news/2002/March03.htm), (Internet, 22nd, january).

(2002c), Framework for engagement with pacific regional nonstate actors. (http://www.forumsec.org.fj/docsGen_Docs/ngo\%20pol.pdf) (Internet, 3rd, july, 2005).

(2003a), Press Statement 98/03: Speakers meet on new secretariat, (http://www. forumsec.org.fj/news/2003/Oct/Oct\%2002.htm (Internet, 2nd, december, 2003).

(2003b), "Forum principles of good leadership", (http://www. forumsec.org.fj/docs/Communique/2003\%20 Communique.pdf), (Internet, 10th October 2006).

(2003c), Twenty-fourth pacific islands forum: forum communique, (http://www. forumsec.org.ff/docs/Communique/2003\%20 Communique.pdf) (Internet, 10th, october, 2006).

(2003d), Press Statement 110/03: Visit of the Forum Review Eminent Persons Group. (http://www.forumsec.org.fj/news/2003/Nov/Nov $\% 20$ 06.htm) (Internet, 12th October 2006).

(2004a) Press Statement 27-04: Statement by the Forum Secretary General, Mr Greg Urwin at the 4th Forum Presiding Officers' Conference. (http://www.forumsec.org. fj/news/2004/Apr/Apr_26.htm) (Internet, 9th, july, 2004).

(2004b) Press Statement 83-04: Secretary general welcomes inputs from regional non-state actors in the design of the pacific plan. (http://www. forumsec.org.fj/news/ 2004/October/Oct_13.htm), (Internet, 24th, november 2004).

(2005) Press Statement 19/05: Opening address by greg urwin, secretary general, pacific islands forum secretariat at the third multi-stakeholders workshop for the pacific islands association of non-governmental organisations 
(PIANGO) on strengthening civil society in the pacific. (http://www.forumsec. org.fj/news/2005/ April/02.htm) (Internet, 25th, may, 2005).

$$
\text { or. } / \text { hews/2005/ April/02.htm) (Internet, 25th, may, 2005). }
$$

-Hilda Lini, Freedom Fighter, "A believer in people oriented governance" Pacific Magazine ( july, 2001).

-Policy on consultative status and accreditation between the pacific islands forum (PIF) and Pacific regional non-state actors (PRNSA) (2007) (http:// www.piango.org/docs_RegMtsg/CSO/CSO2007_Forum_communique.pdf) (Internet, 3rd, march, 2008).

-Reilly, Benjamin (2004), "State functioning and State failure in the south pacific", Australian Journal of International Affairs, 58 (4).

-Reilly, Benjamin and Wainwright, Elsina (2005), “The South Pacific”, en: Simon Chesterman, Michael Ignatieff and Ramesh Thakur (eds.), Making States work: State failure and the crisis of governance. Tokyo, United Nations University Press. tement".

-South pacific forum secretariat (1995), "South pacific forum vision sta(1) tion". (1997a), "Aitutaki declaration on regional security coopera, (1997b), "Forum economic ministers meeting action plan". , (1998), "Forum Economic Action Plan, 1998 Review".

- Thistlethsaite, Bob and Davis, Derrin (1996), Pacific 2010: A sustainable future for melanesia? Natural resources, population and development, Canberra, National Centre for Development Studies, Australian National University. 
